\title{
Physical and Optical Properties of
}

Rare Earth Cobalt Magnets

\author{
Klaus Halbach \\ Lawrence Berkeley Laboratory, UC Berkeley, \\ Berkeley, CA. 94720
}

Rare Earth Cobalt (REC) permanent magnets have unique properties that permit solutions to sone optical tasks that cannot be accomplished with conventional magnets. A review of design and of performance characteristics of these magnets includes an analytical description of the three dimension 1 fringe fields of REC quadrupoles.

\section{1) Introduction}

There are indications that Rare Earth Cobalt (REC) permanent magnets will soon be used much more frequently to solve optical problems that cannot be solved with conventional means. It is the purpose of this paper to summarize information that is usefui for the design of REC magnets and 0 assess their performance characteristics. The optical properties of the devices di cussed here usually can be obtained directly and very simply from the magnetic field distributions. The emphasis is therefore on the description of the lat:er, the former following in most cases directiy by implication.

Since REC magnets have not yet been used extensively, the choice of devices that are discussed in detail reflects my expectation of which $k$ ind of REC magnet will become important in the near future.

Design and performance formulas are given to allow the reader to make a decision whether a REC device is a good choice for his needs. The specific designs of magnets that are discussed represent a good compromise between performance and cost for most applications. If the reader wants to work out more details of a particular magnet, he may find Ref. 1 useful. That paper gives general design philosophy and procedures for the design of REC magnets, and extensive details about the design of two dimensional (20) multipoles. Quantitative details about the fringe fields of quadrupoles as well as a description of the other devices discussed in this paper will appear in future publications by the author.

\section{2) Kotation}

MKS units are used throughout, with $\mu_{0}=4 \mathrm{~m} \times 10^{-7}$ Vsec $\mathrm{A}^{-1} \mathrm{~m}^{-1}$. For 2D fields it is convenient to express fields by analytical functions of a complex variable. Complex quantities are identified by underlining. Specifically, $z$ is one of the three space coordinates, but $z=x+i y .2 D$ fields are described by $B=B_{x}+i B_{y}$, with an asteris!* indicating the complex conjugate of a complex number. Br indicates the magnitude of the remanent magnetization of the material, and $B_{r}$ is the 20 remanent magnetization vector. 


\section{3) Material Properties}

The development of REC materials started in 1966 with Strnat's (2) work. Brief sumartes of the manufacturing process cin besfound in References 1 and 3 , wille Ref. 4 goes into detail. He give here only a brief description of the properties of comarcially available REC.

Oriented REC material is a agneticaliy anisotrópic material with a strong intirinsic manetization in the drection of a preferred crystalline axis, conimion called the easy axis. Fig. 1 shows the relationship between the fields $B_{11}$ and ${ }_{0} H_{1}$ in the direction parallel to that easy axis fhis $\mathrm{B}_{\text {, }}\left(\mu_{0} \mathrm{H}_{f l}\right)$-curve is for all intents and purposes, straight line in the first quadrant and in a substantial part of the second, or even third, quadrant. The slope of the curve in the straight part of the curve is typically dB $/ \mathrm{d}$ ( $\mathrm{HH} / \mathrm{H}$ ) $=1.04$, and the remanent field $\mathrm{B}_{r}$ is usually in the range $.85-1.05 \mathrm{~T}$. The locition of the point where the slope of the $B_{\| \prime}\left(\mu_{0} H_{\|}\right)$-curve increases significantly, $i . e_{.}$, the knee of the $\mathrm{B}_{\|}\left(\mathrm{\mu}_{0} \mathrm{H}_{\|}\right)$-curve, depends on Danufacturing details and cannot be modified by the user. For readily available materials, the knee is located less deeply in the second or third quadrant for larger values of $B_{r}$. The working point can be moved neversibiy along the straight part of the $B_{\|}\left(\mu_{0} H_{n}\right)$-curye, but when one noves into or beyond the kmee, the recoil will occur along a straight line parallel to the initial straight part of the $\mathrm{B}_{\|}\left(\mu_{0} \mathrm{H}_{\|}\right)$-curve, and the initial magnetization can be recovered only by driving the material very far into the first quadrant.

The $\mathrm{B}_{4}\left(\mathrm{moH}_{4}\right)$-curve in any direction perpendicular to the easy asis is a straight tine through the origin $\mathrm{B}_{L}=\mu_{0} \mathrm{H}_{1}=0$, with a slope very. simitar to the slope of the $\mathrm{B}_{H}\left(\mu_{0} \mathrm{H}_{\|}\right)$-curve. All formulas given in this paper have been derived with the simplifying assumption that the slopes of both curves are one. Tie most important consequence of this assumption is the applicability of the linear superposition of vacuim fields. In real life, this assumption is violated only very slightly, particularly because the fost damaging deviations from vacuum fields are sensitive only to the difference in differential permeability in the directions parallel and perpendicular to the easy axis.

For the sake of completeness, it should be pointed out that some ferrites behave qualitatively similar to REC, but are quantitatively different: the differential permeabilities are $\geq 1.1$ and $B_{r}$ is only $.2-.35 \mathrm{~T}$.

It is difficuit to talk abouc the price of finished pieces of magnetized REC because many different variabies entor, like magnetic characteristics of the material, size and shape of pieces, tolerances of dinensions and magnetic properties, total volume of order, etc. But to give a rough guideline for pricing $_{3}$ it can be said that most comercial orders will cost between 1.5 and $30 \mathrm{~s} / \mathrm{cm}^{3}$. Ferrites are substantially less expensive.

\section{4) General Properties of REC Alagnets for Particle Optics Applications}

This section presents a muber of facts that are applicable to all REC agnets. These facts are often compared with equivalent properties of comentional megnets to give an indication under mat circusstences the REC anger would be preferable. 


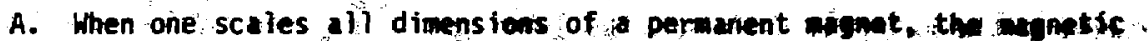
fields do not change': then one reduces the size of a conventional thents and wants the fields to remin constant, the current density in the coils must increase. At some size this will lead to insurmountabs aling problems that force a reduction of the field strength. Conseguently, a REC magnet can always produce higher fields belowa certatn siz of the magnet or working volume. : To avoid a misinterpretation of tils statent, it has to be added that for some types of magnets, the REC menet can made stronger than the equivalent conventional magnet, regardless of size.

B. REC magnets are usually quite compact and light, and obviously do not require power supplies or cooling. These properties are of great importance in some applications, like drift tube quadrupoles in a LINAC, or spectrometers in satelilites or rockets.

C. The absence of power supply leads and plunbing associated with either conventional or He cooling makes REC magnets very attractive for some applications, for example a variable gap undulator inside a vacuum envelope:

D. The applicability of linear superposition of magnetic fields makes analytical description of REC magnets very easy. I consider this quite important because analytical treatment nearly always leads to good understanding, from which good design and innovation nearly always flow. The validity of linear superposition of fields also leads to a number of remarkable and unusual configurations and system properties, some of which are discussed below in subsections $E$ and $F$.

E. REC magnets can be placed inside other magnets, with linear superposition of fields in the common working volume, and very little magnetic interference in the rest of the magnetic field volume. Typical anticipated applications are: a) A strong final focussing REC quadrupole inside the solenoid of a storage ring or single pass collider interaction region detector. b) A REC undulator with weak quadrupole windings. c) A REC quadrupole inside the working volume of another REC quadrupole, giving a variable strength quadrupole when the two quadrupoles are rotated relative to each other.

F. Because of the superposition principle, most pure REC magnets produce fields that are very small outside the working volume as well as the magnet itself, and decay there over very short distances.

G. There is not very wuch known about the behayiour of REC magnets in a high radiation environment. Materials experts do not believe that the magnetic properties deteriorate easily. Scme preliminary work confins this, and detafled work will comence in the mear future.

H. REC magnets can easily be taken to $200^{\circ} \mathrm{C}$, and oven higher temperaturas if proper precautions are taken. However, the tencerature should always be fairly uniform and, for that reason, should not be changed too rapidiy.

1. With the presently most widely used andecturing tochnteres, the sinallest dinension of a ReC block cannot be larger then a few centimeters. Even though mamets cal be (end are) assembled from any blecks, devices invotving large wolwes of Re become very expensive 
becucte of the mater tel costs and the labor costs involved in making and ining ing fary large number of indivitual components.

\section{5) Muttipole Maghets}

To oroduce strong 20 mittipole with sed fielo quality (i.e, a REC magat inth $8^{*}-2^{-1}$ ) the REC ideally should fill the area between two concenitrie circlet, and the easy axis of the nterial at the polar coordinate location $r$ s shoijd form the angle

$$
\text { of })=(n+1)+1
$$

with the $=0$ direction.

Since it would be very difficult to produce such a magnet, one has to make a compromise betiven performance and cost of the magnet. The following th practical design is such a compromise: Break up the material between the concentric circle's into $A$ geometrically. identical blocks. With in each block the easy axis has the same orientation, and that orientation is given by eqn. (1), with now identifying a fiducial mark on the block. Fig. 2 shows a schematic cross-section of such a multipole magnet (in this case a quadrupole, i.e.. $N-2$ ), with the arrows inside the blocks indicating the directions of the easy axes.

If the individual blocks are touching trapezoids, the 20 fields produced by a $2 \mathrm{~N}$-pole magnet inside the Working aperture is given by

$$
\begin{aligned}
& B^{\prime}\left(z_{0}\right)=B_{r} \cdot \sum_{r=0}\left(\frac{z_{0}}{r_{1}}\right)^{n-1} \cdot \frac{n}{n-1} \cdot\left[1-\left(\frac{r_{1}}{r_{2}}\right)^{n-1}\right] \cdot C_{n} \\
& n=N+V \cdot M \\
& C_{n}=\cos ^{n}(\pi / M) \cdot \sin (n \pi / M) /(n \pi / M) \\
& \left(\frac{n}{n-1}\left[1-\left(\frac{\gamma_{1}}{\gamma_{2}}\right)^{n-1}\right]\right)_{n \rightarrow 1}=\ln \left(\gamma_{2} / \gamma_{1}\right)
\end{aligned}
$$

Br is the complex representation of the menetization vector of the reference trapezoidal block that is bisected by the positive $x$-axis; $r_{1}$ and $r_{2}$ are the distances between the courdiante origin and the intersections between the neference block and the pesitive $x$-axis; and $M$ is the aumer of trapezolel blocks pre magnet. Ref. I gives also the formulas for muitipoles with differently shaped or arranged blocks. They are structurally identical to Eqn. (2). but with ifferent expressions $C_{n}$. 


$$
\left.\begin{array}{l}
B^{*}\left(z_{0}\right)=B_{r} \cdot \sum_{r=1}\left(\frac{r_{2}}{\underline{z}_{0}}\right)^{n+1} \cdot \frac{n}{n+1} \cdot\left[1-\left(\frac{r_{1}}{r_{2}}\right)^{n+1}\right] \cdot C_{-n} \\
n=V \cdot M-N \\
C_{-n}=\cos ^{-n}(\pi, M) \cdot \sin (n \pi / M) /(n \pi / M)
\end{array}\right\}(3)
$$

From Eqn's. (2) and (3) follow three important facts:

$A$. The segnentation of the magnet, $i . e .$, the finite magnitude of $M$, leads to a reduced mignetic field (expressed by $C_{n}$ ) and to harmonics that may be harmfui. For quadrupole $(N=2)$ with $M=16, C_{2}=.94$ arid the first two undesired harmonics are $n=18$ and $n=34$. Clearly, $c_{2}$ is adequately close to the ideal value 1. In the unlikely case that the first undesired harmonic is bothersome, its amplitude can be made zero by separating the REC blocks by thin non-magnetic shims according to a prescription given in Ref. 1.

B. The segnentation of the omultipole leads also to non-zero fields outside the magnet. However, for reasonably large values of $M$ these fields are so small and decay so rapidly that they are rarely of concern.

C. The field strength of the fundamental at the multipole aperture

$$
\left(B_{r} \cdot \frac{N}{N-1} \cdot\left[1-\left(\frac{r_{1}}{r_{2}}\right)^{N-1}\right] C_{N}\right) \text { can, for low order multipoles like dipoles }
$$

and quadrupoles, exceed $B_{r}$ significantly. It has to be remembered that this field strength is attained only if the material under these operating conditions is not driven into or beyond the knee of the $B$ " ( $\left.\mu_{0} H_{1 f}\right)$-curve. Some materials are comercially available that allow aperture fields of $1.2 \mathrm{~T}$.

In addition, if the ends of a multipole are cut off with two planes perpendicular io the magnet axis, the following statements are true:

D. The effective magnetic length equals its physical length.

E. Higher harmonics that are not present in the 2D cross-section are not present in the fringe field region either. In this context, higher harmonics are identified by the dependence of fields or potentials on $\$$

6) Additional Detalls for Quadrupoles and Dipoles.

A. If one replaces all REC inside a closed scalar potential surface by high permedblitity steel, the performance of the magnet will not be affected as long as the perneability of the steel is large enough. One can find such closed scalar potential surfaces for witfpoles, but the savings in REC materials cost is usually not worth the conplication in constevctien, except possibly for a dipole. When a dpole is meeded with a working volus wose 
cross-section much wider than it is high, this technique can lead to a rather attractive design. However, detailed design studies ad comparison with conventional combined REC-steel magnet designs have not yet been done.

B. Quadrupoles of the basic design shown in Fig. 2 have been built in large numbers by R. F. Holsinger. They have all the expected properties. Details of design and measurementis of these quadrupuies $\leq \geq n$ be round in Ref. 5 and 6 .

C. It is often desirable to adjust the strength of quadrupoles. This can be done in at least three different ways with steel-free REC magnets. a) As mentioned in Sect. $4 E C$, one segmented quadrupole (or a quadrupole assent led from rods of circular cross-section, as proposed in Ref. 7) could be placed inside the working volume of a larger quadrupole of the same design. If both quadrupoles produce the same field gradient, the combined gradient can be changed without changing the quadrupole ield orientation if the two. quadrupoles are rotated by the same anciant in oppasite directions. However, since the end-fringe fields from the two quadrupoles behave differentily, the fields in the end regiens will change direction. (See Sect. $6 \mathrm{0.}$ ) This in turn will lead to some optical coupling between the usually decoupled two major planes of a quadrupole. b) To avoid this dramback, one can build a. segmented quadrupole with radially movable segments. The trouble with this design is its mechanical complexity. c) One can also break up a quadrupole into a number of axial "slices" and rotate them individually in such a manner that the optical strength of the system is modified wtthout introducing the coupling associated with the method described in Sect. 6 Ca. R. L. Gluckstern has studied this problem, found a solution, and will present it at this Conference $(8)$.

D. The fringe field of a quadrupole can be of interest for a number of reasons, for instance to assess the coupling introduced by an adjustable quadrupole system of the kind described in Sect. $6 \mathrm{Ca}$, or to obtain information about optical aberrations. Since the higher harmonics are usually of such high order that their contribution to the fringe fields is of no interest, only the fringe field of the fundimental is described here. The configuration considered here is a semi-infinite quadrupole with a flatly cut end, as show in Fig. 3. The fringe field can be derived from the scalar potential $v(x, y, z)$, given by the following equations:

$$
\begin{aligned}
& V(x, y, z)=B_{r} \cdot C_{2} \cdot\left(\frac{1}{r_{1}}-\frac{1}{r_{2}}\right) \cdot\left(x^{2}-y^{2}\right) \cdot L_{2}\left[\left(x^{2}+y^{2}\right) \cdot \frac{\partial^{2}}{\partial z^{2}}\right] \cdot F(z) \quad \text { (4a) } \\
& L_{2}(u)=\sum_{r=0} \frac{2 \cdot(-u / 4)}{v !(v+2) !}=1-\frac{u}{12}+\cdots \\
& v_{2}=1 / \sqrt{1+\left(z / r_{2}\right)^{2}}
\end{aligned}
$$$$
F(z)=\frac{1}{2} \cdot\left(1-\frac{\frac{1}{v_{1}}+\frac{1}{v_{2}}}{8} \cdot z \cdot \frac{v_{1}^{2} v_{2}^{2}\left(v_{1}^{2}+v_{1} v_{2}+v_{2}^{2}+4+8 / v_{1} v_{2}\right)}{v_{1}+v_{2}}\right)
$$ 


$$
F^{\prime \prime}(z)=\frac{3}{8} \frac{\left(\frac{1}{r_{1}}-\frac{1}{r_{2}}\right)^{-1}}{z^{3}}\left(\left(1+\frac{y}{2} z^{2} / r_{2}^{2}\right) v_{2}^{y}-\left(1+\frac{\gamma}{2} z^{2} / r_{1}^{2}\right) v_{1}^{y}\right) \quad(4 e)
$$

These formulas are valid when the quadrupole segments fill tire space between two circles with radii $r_{1}$ and $r_{2}$ completely. In this case,

$$
C_{1}=\sin (3 \pi / M) /(3 \pi / M):
$$

However, if $M$ is reasonably large, Eqn's. (4) adequately describe the case of a quadrupole assembled from trapezoidal blocks.

Notice that the coordinate system used here is rotated by $45^{\circ}$ relative to tre system normally used to describe quadrupoles.

F" (z) (Eqn. 4e) describes the strength of the lowest order aberration associated with the varying quadrupole strength. Plots of $F$ and $F$ " are reproduced in Figures 4 and 5 for $z \geq 0$. The values of $F$ and $F "$ for $z<0$ follow from the obvious symetry properties of $F$ and $F "$. If one expands $F$ for $z$ » $r_{2}$, the dominant term is given by

$$
F=\frac{1}{32} \cdot \frac{r_{2}^{5}-r_{1}^{5}}{r_{2}-r_{1}}=\frac{r_{1} r_{2}}{2^{6}}
$$

indicating a pleasantly fast decay.

of the many different uses of Eqn's. (4), one deserves special notice. The integrals involved for calculation of the kick received by a particle going in a straight line through the fringe field region are very simple, and cne obtains, with a' wield gradient deep inside the quadrupole:

$$
\int_{-\infty}^{\infty} \Delta B_{x} d z=\frac{B^{\prime}}{6} \int_{-\infty}^{\infty}\left(x_{0}+x_{0}^{\prime} z\right)^{3} \cdot F^{\prime \prime}(z) d z=B^{\prime} \cdot\left(x_{0}^{2} x_{c}^{\prime} / 2+x_{0}^{13} \gamma_{1} r_{2} / 8\right)
$$

In this, equation, the genesis of the existing and non-existing terms is as follows: a term proportional to $x_{0}^{3}$ is always absent; the ten proporticnal to $x_{0}$ is cosent because $F^{\prime \prime}(-z)=-F^{\prime}(z)$; the coefficient of the term proportional to $x_{0}$ " is independent of the detaijed behavior of 
$F(z)$; only the coefficient of the term proportionai to $x_{0}{ }^{3}$ depends on the shape of $F(z)$.

\section{7) Linear Undulators}

Linear undulators can be essential components of free electron lasers, and can be used to produce synchrotron radiation from an electron beam, or even a proton beam (for beam diagnostics $(9)$ purposes). Under some circumstances, REC undulators are preferable to conventional ones, and Ref. 10 describes the use of a REC undulator in an optical clystron, but without giving any of the undulator design details.

Fig. 6 shows a schematic cross-section of a linear undulator with period length $\lambda$ and $M^{\prime}=4$ blocks of REC per period in each half of the undulator. If the undulator is long enough in the direction perpendicular to the paper plane, the purely 20 field inside the undulator $(i . e .,-h<y<h$ ) is given by

$$
\left.\begin{array}{l}
\underline{B}^{*}(\underline{z})=i \cdot \lambda \cdot B_{r} \cdot \sum_{V=0} \cos (n k z) \cdot e^{-n k h} \cdot\left(1-e^{-n k L}\right) \sin \left(n \varepsilon \pi / M^{\prime}\right) /\left(n \pi / M^{\prime}\right) \\
n=1+\left.v \cdot M\right|^{\prime} ; k=2 \pi / \lambda
\end{array}\right\}(5)
$$

It has been assumed that from each block to the next, the easy axis is rotated by $2 \pi / M^{\prime}$.

The structure of this equation is very similar to the structure of the equation describing the field of a segmented multipole: Because of the segnentation, there are harmonics present; as in the case of multipoles, the harmonics corresponding to negative $n$ lead to fields outside the device (i.e., $|y|>h+L$ ): but are rat given here. For most practical values of $k h$, the higher harmonics are not of great importance. The first of the harmonics can be cancelled by u'sing $c=1 /\left(1+1 / M^{\prime}\right)$. However, there is. a loss of amplitudes of the fundamental involved (which can be reduced with a more complicated shape of the blocks), so that one would use this procedure only when absolutely frecessary.

If one ignores the synchrotron radiation effects, the effect of the undulator fields on particle trajectories is, to lowest order in $\left(B_{\max } / k\right) /(p / e)\left(B_{\max }=\max i \operatorname{mum}\right.$ field for $y=0 ; p$ and $e$ are momentum and charge of the particle), as follows: in the $z$ direction, the device acts for all intents and purposes like a pure drift space, and the motion in the $y$ direction is governed by

$$
y^{\prime \prime}+y \cdot\left(B_{\max } e / 0\right)^{2} / 2=0 \text {. }
$$

Eqn. (5) shows the sane hard liait on the achievable field strength that charaterizes all pure REC magnets: for gtven geometric parameters. the cbtainablefefid strenth is Jinited by the properties of the REC aterial. For this reason, high field undlators with Jarge geps and perfod lengths should be built with comientional technoley. Howerer, for nost of the presently interestim cem inations of sountic paremters and field strength, the REC undulator is the ideal solution. If one wishes to have an undulator 
with a small period length and a higher field than is obtainable with a REC undulator, one has to consider a superconducting undulatory. Unfortuantely. the cost and operational complexity of such a device are often so prohibitive that one has to be content with the performance of the REC undulatory.

It is of ten important to adjust the fields at the entrance and exit of the undulator in such a way that the total flux traversed by the bean is zero and a field symmetry condition is accurately satisfied. These adjustments can be done either by mechanically rotating REC blocks at the ends or by energizing tuning coils. These systems are too specific to be included here.

In the construction of an undulatory it is important to make the device long enough in the $z$ direction of Fig. 6 so that the finite length of the device does not modify significantly the $2 D$ fields seen by the beam. On the other hand, for cost and other reasons one does not want to make the undulator wider than necessary.

To provide the information needed to determine the proper width of an undulatory, the following En's. (6) can be used to evaluate By in the midplane of a semi-inf incite undulatory (as shown in Fig. 7), thereby permitting the calculation of the effect of the "missing" parts of an undulatory of infinite width.

$$
\begin{aligned}
& \Delta_{1}=k h ; B_{2}=k(h+L) ; r=k z ; p=\sqrt{a^{2}+r^{2}} \\
& \delta=\gamma \cdot\left(\sqrt{1+\gamma^{2} / \beta^{2}}+1\right)^{-1 / 2} / \beta \\
& B_{c}=2 \cdot B_{r} \cdot \sin \left(\varepsilon \pi / M^{\prime}\right) /\left(\pi / M^{\prime}\right) \\
& B_{y}(z)=\beta_{0} \cdot\left(U\left(\beta_{1}, \gamma\right)-U\left(\beta_{2}, \gamma\right)\right) \\
& U(\beta, \gamma)=e^{-\beta} \cdot\left(\sqrt{1+\gamma^{2} / \beta^{2}}+1\right)^{1 / 2} \cdot W(\beta, \gamma) \\
& h(\beta, Y)=\frac{1}{\pi} \cdot \int_{0}^{x} \frac{e \lambda p(-e u /(1-u))}{\sqrt{2-u}} \cdot \frac{d x}{i+2 x(x-1)} \\
& a=\delta \cdot(1 x-1))_{i}^{2} x_{1}=1 /(1+\delta)
\end{aligned}
$$

Information for $\gamma<0$ is obtainable from the behaviour for $Y>0$ through

$$
U(\beta,-\gamma)=e^{-\rho}-(U(\beta, \gamma)
$$


The "output" information is obtained from Eqn. (6d), with Eqn's. (6e) and (6f) providing the essential inputifor Eqn. (6d). Instead of a graph of $U(s, \gamma)$. Fig. 8 shows the less violently changing function $U(\beta, \gamma) \cdot e^{p}$.

8. Helical Undulator

Under some circuristances it is more advantageous to use a helical undulator, i.e., a device that produces a field that has on axis a dipole component in the direction perpendicular to the axis, with the direction of that field changing uniformly as one moves along the axis uniformly.

To implement sugh an undulator with REC, one can build short (in the 2-direction) segmented dipoles, and rotate each short dipole relative to the previous dipole so that one obtains the desired period length $\lambda$ in the $z$ direction. If there are $N^{\prime}$ such slices per period $\lambda$, one obtains for the strength, on axis, of this REC unduiator

$$
\begin{aligned}
& B=B_{r}: C_{1}, \frac{\sin \pi / N^{\prime}}{\pi / N^{\prime}}=\left(T\left(x_{1}\right)-T\left(x_{2}\right)\right) \\
& T(x)=K_{0}(x)+\frac{x}{2} \cdot K_{1}(x) \\
& x=2 \pi r / \lambda .
\end{aligned}
$$

Eqn. (7a) is valid for the case where the segments fill the space between two circles with radii $r_{1}$ and $r_{2}$ completely, and

$$
C_{1}=\frac{\sin 2 \pi / M}{2 \pi / \pi}
$$

with $M=$ number of segments in a dipole slice. However, these equations can be applied to the design with trapezoidal segments with very little error when $M$ is reasonably large.

$K_{0}$ and $K_{1}$ in Eqn. (7b) are modified Bessel functions, and a graphic representation of $\ln (T(x))$ is reproduced in Fig. 9 .

\section{9) Production of Solemidal Fields with REC}

It is c?ear that sowe properties of fields prodused by solenoids are impossible to reproduce with REC structures. The most obvious condition that is a lwass satisfied by REC devices that produce fields of the same symatry as solenoits is

$$
\int_{-\infty}^{\infty} \mathrm{B}_{z}(z) d z=0 \text {. }
$$


While the same integral is in general not zero when solenoids produce the fields, Eqn. (8) is not a severe restriction under many circumstances, since matrix elements $m(z)$ of the first order optical transfer matrices of a bean travelling along the axis satisfy the differential equation

$$
\begin{aligned}
& m(z)^{\prime \prime}+k^{2} \cdot m(z)=0 \\
& k(z)=\frac{B_{z}(z)}{2 p / e}
\end{aligned}
$$

In Eqn. (9), $p$ and $e$ are momentum and charge of the particle, and it is assumed that the matrix is expressed in a coordinate system that is rotated by

$$
a(z)=-\int_{z \text { start }}^{z} k(z) d z
$$

relative to its orientation at the starting point. Since it is $k^{2}$ that appears in Eqn. (9), the sign reversals of $B_{z}(z)$ are usually not of major importance.

To give a feeling of what one can accomplish with REC, consider a segmented periodic array of magnetized REC rings, as shown in Fig. 10 . With $M^{\prime}$ rings per period $\lambda$, and an easy axis rotation of $2 \pi / M^{\prime}$ from one ring to the next, one obtains for the scalar potential

$$
V=-B_{r} \cdot \sum_{v=0} \frac{\sin (n k z)}{n k} \cdot I_{0}(k r) \cdot\left(G\left(n k_{1}\right)-G\left(n k_{2}\right) \cdot \frac{\sin n \pi / M^{\prime}}{n x / A^{\prime}}\right.
$$

and for the field on axis

$$
\left.\left.B_{2} i \geq\right)=B_{r} \cdot \sum_{i=0} \cos (n k z) \cdot\left(G\left(n x_{1}\right)-G\left(n x_{2}\right)\right) \cdot \sin (n \pi / M) /(n \pi / M 1)(1) 6\right)
$$

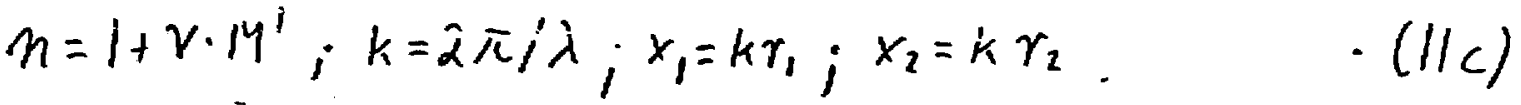

$$
\begin{aligned}
& G(x)=x \cdot K_{1}(x)+K_{0}(x)+\int_{x}^{\infty} K_{0}(x) d x
\end{aligned}
$$


In Eqn's. (11a) and (ild), $I_{0}, K_{0}$, and $K_{1}$ are modified Bessel

functions. They, as weli as the integral in Eqn. (11d), are tabulated in Ref. 11. A graphical representation of Equ. (11d) is given in Fig. 11. It should be noted agsin that the asymptotic amplitude of $(1+\pi / 2) \cdot B_{r}$ for $B(z)$ cannot be reached tmless the mat ria? has a knee sufficiently far in the third quadrant of the $\mathrm{B}_{n}\left(\mathrm{nO}_{n} \mathrm{H}_{n}\right)$-curve.

\section{REFERENCES}

1. K. Hálbach, NIM 169 (1980); 1

2) K.J. Strnat and G.I... ioffer, Techn. Report, AFM-TR-65-446, Wright Paterson Air Force Base, (1966); J.B.Y. Tsui, D.J. Iden. K.J. Strnat and A.J. Evers, IEEE Trans. Magin. 2 (1972) p. 188.

3) K. Halbach, Proc. 1979 Particle Accelerator Conf.. IEEE Trans. Nuc1. Sci. NS-26 (1379) 3882 .

4) M. McCaig, Permanent Magnets in theory and practice. (J. Wiley, London, $1977)$.

5) R.F. Holsinger and K. Halbach, Proc. 4 International Workshop on Rare Earth Cobalt Permanent Magnets (1979) p. 37.

6) R.F. Holsinger; Proc. of 1979 Linear Acce1. Conf., Brookhaven, BNL-51134, p. 373.

7) N.V. Lazarev, S. Skachkov; .Proc. of 1979 Linear Acce1. Conf., Brookhaven, BNL-51134, p. 380 .

8) R.L. Gluckstern, Proc. of this Conference.

9) R. Coisson; IEEE Transactions Vol. 24, No. 3, June 1977, p. 1581.

10) Unpublished internal report. A.S. Artamanov, et.al., Institute for Niuclear Physics, Novosibirsk.

11) Handbook of Mathematical Functions, edited by M. Abramowitz and I.A. Stegun. MBS Applied Mathematics Series No. 55; (1964).

$\star$ This work was supported by the U.S. Dept. of Energy under contract W-7405-EMG-48. 


\section{FIGLRE CAPTIONS}

Figure $]-B_{\| \prime}\left(\mu_{0} H_{i l}\right)$-curve for REC.

Figure 2 - 20 cross-section of segmented REG quadrupole (beam in draming plane).

Figure 3 - Cross-section through semi-infinite REC quadrupole (beam in drawing plane).

Figure 4 - REC quadrupole fringe field strength.

Figure 5 - Second derivative of REC quadrupole fringe field strength.

Figure 6 - 20 cross-section of linear REC undulator (beam in drawing plane).

Figure 7 - Cross-section trirough semi-inf inite REC undulator (beam perpendicular to drawing pline).

Figure 8 - Fringe field strength of REC undulator.

Figure 9 - Strength of helical REC, undulator.

Figure 10 - Periodic array or REC rings (beam in drawing plans).

Figure 11 - Field strength on axis of periodic array of REC rings. 


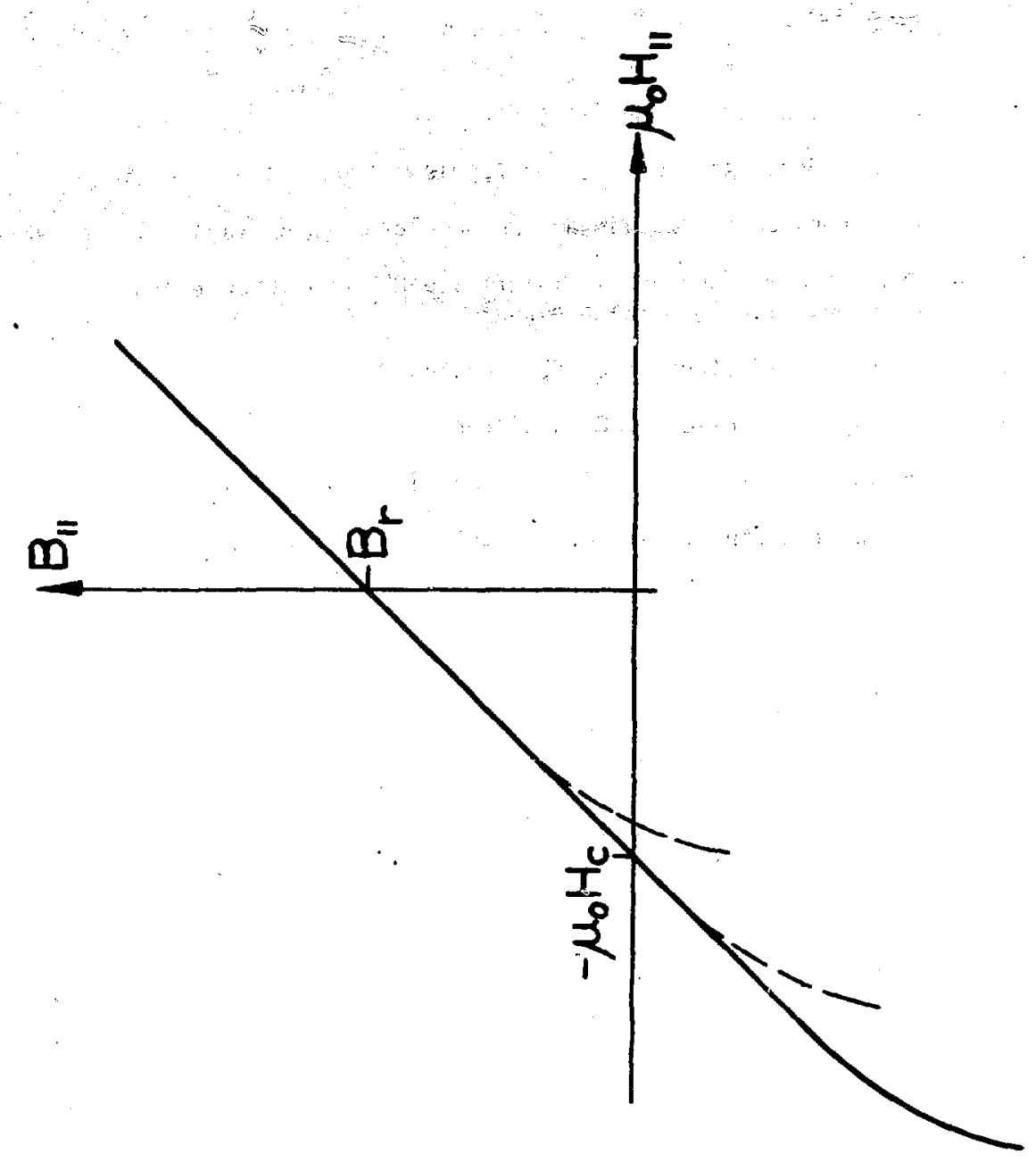

高 


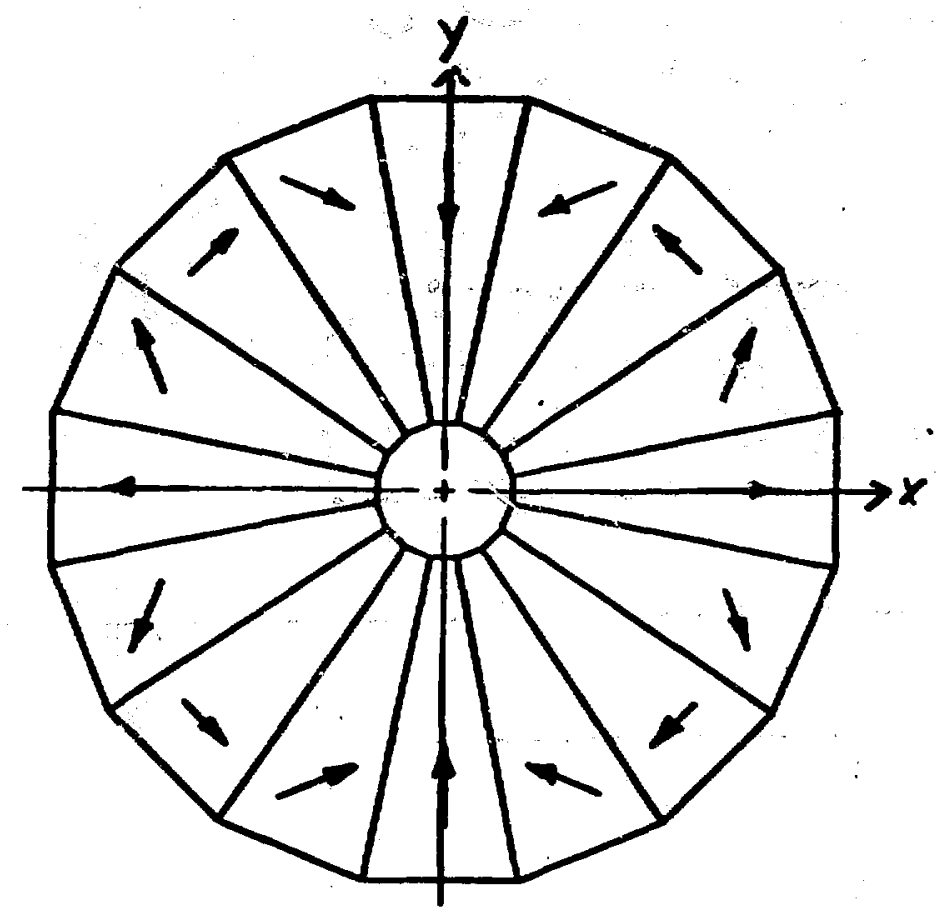

Figure 2 

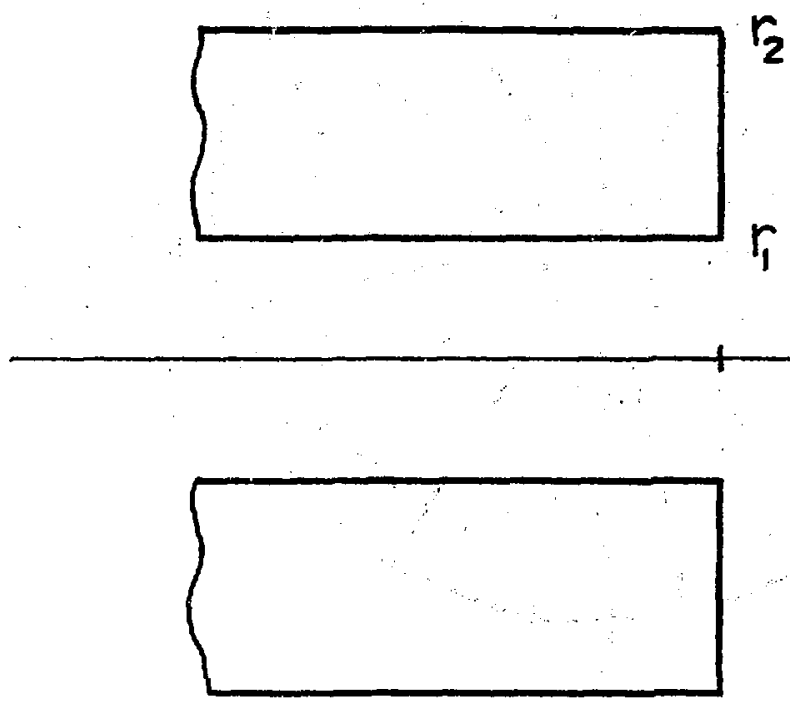

Figure 3 


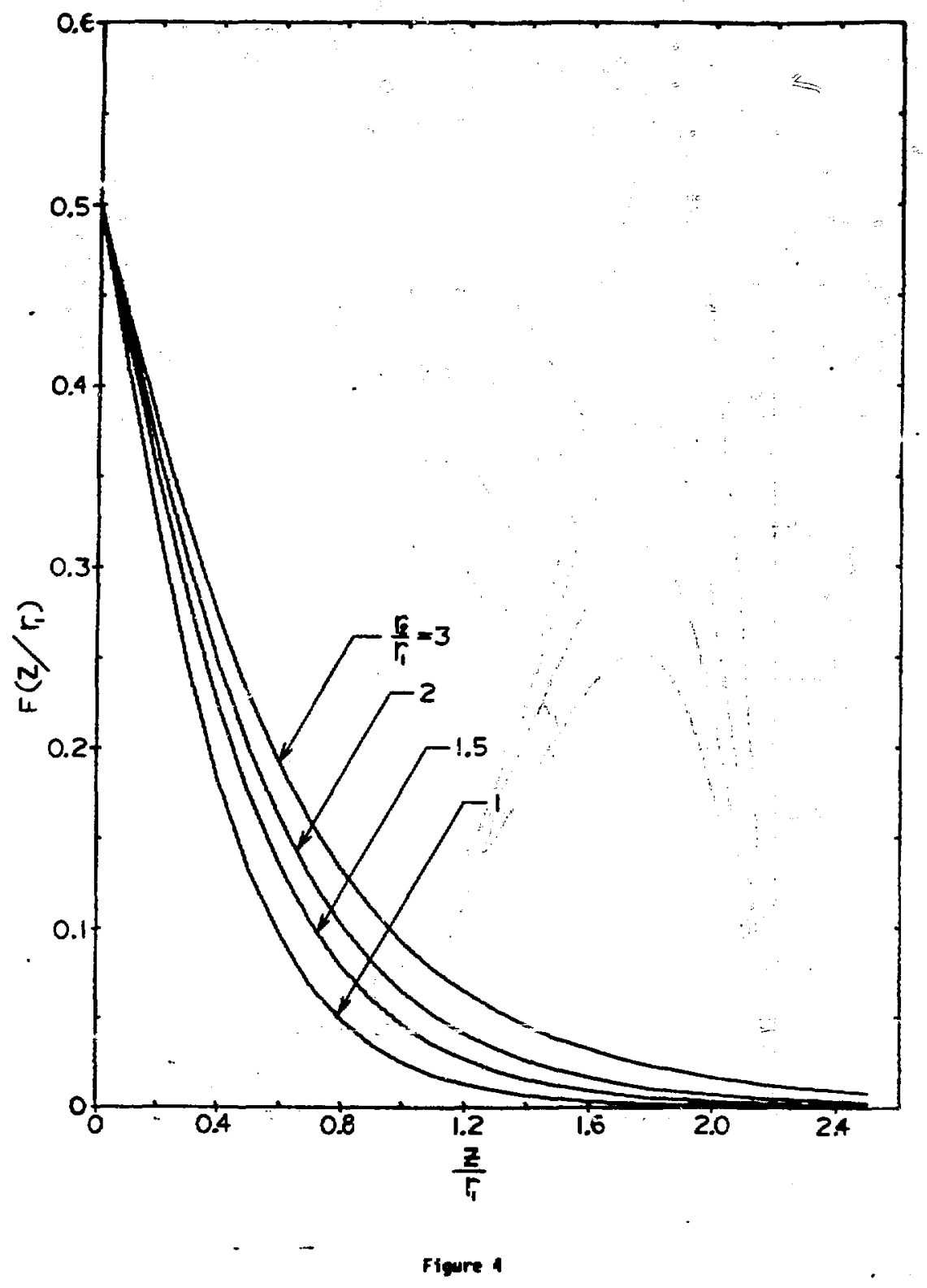

17 


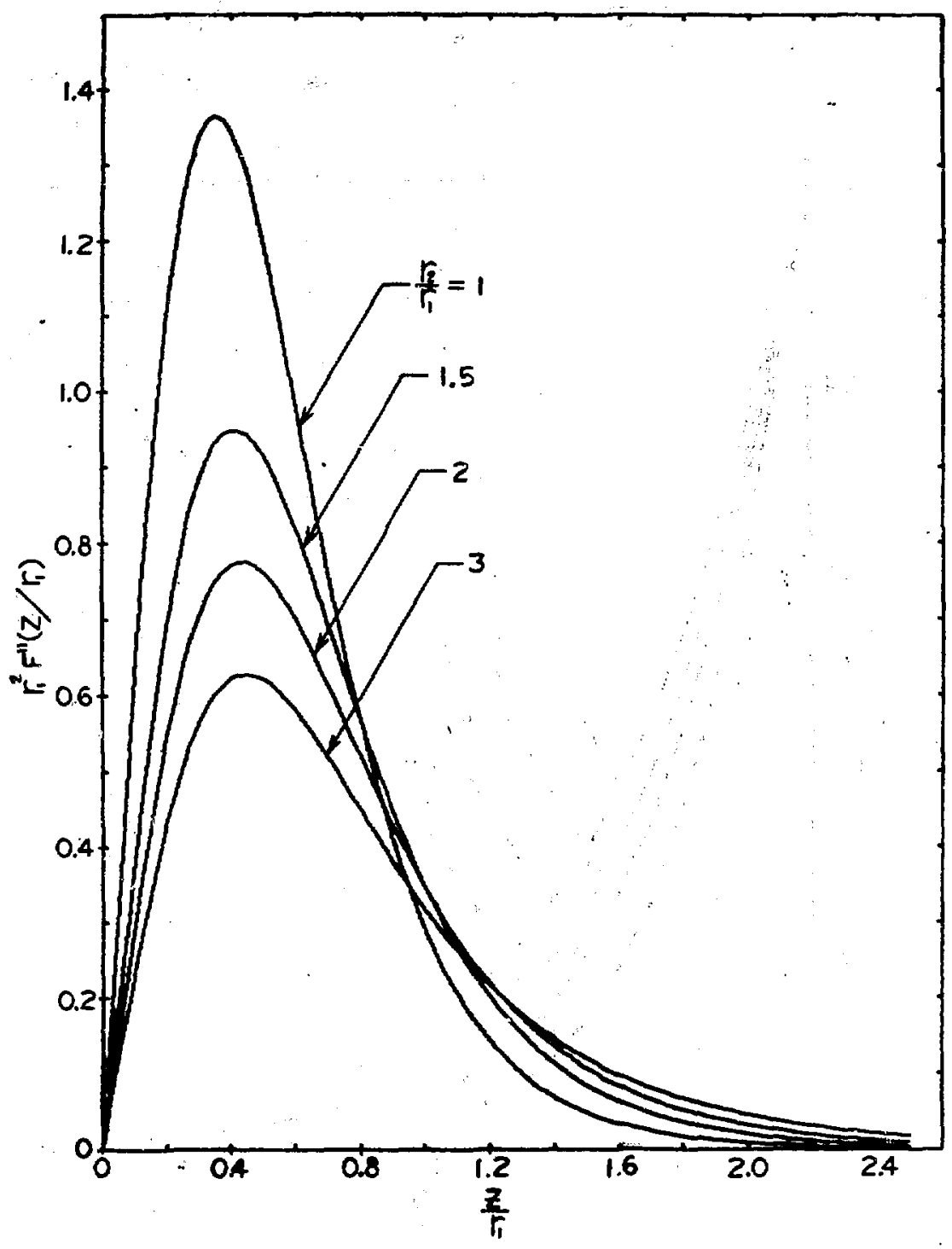

Fisure 5 


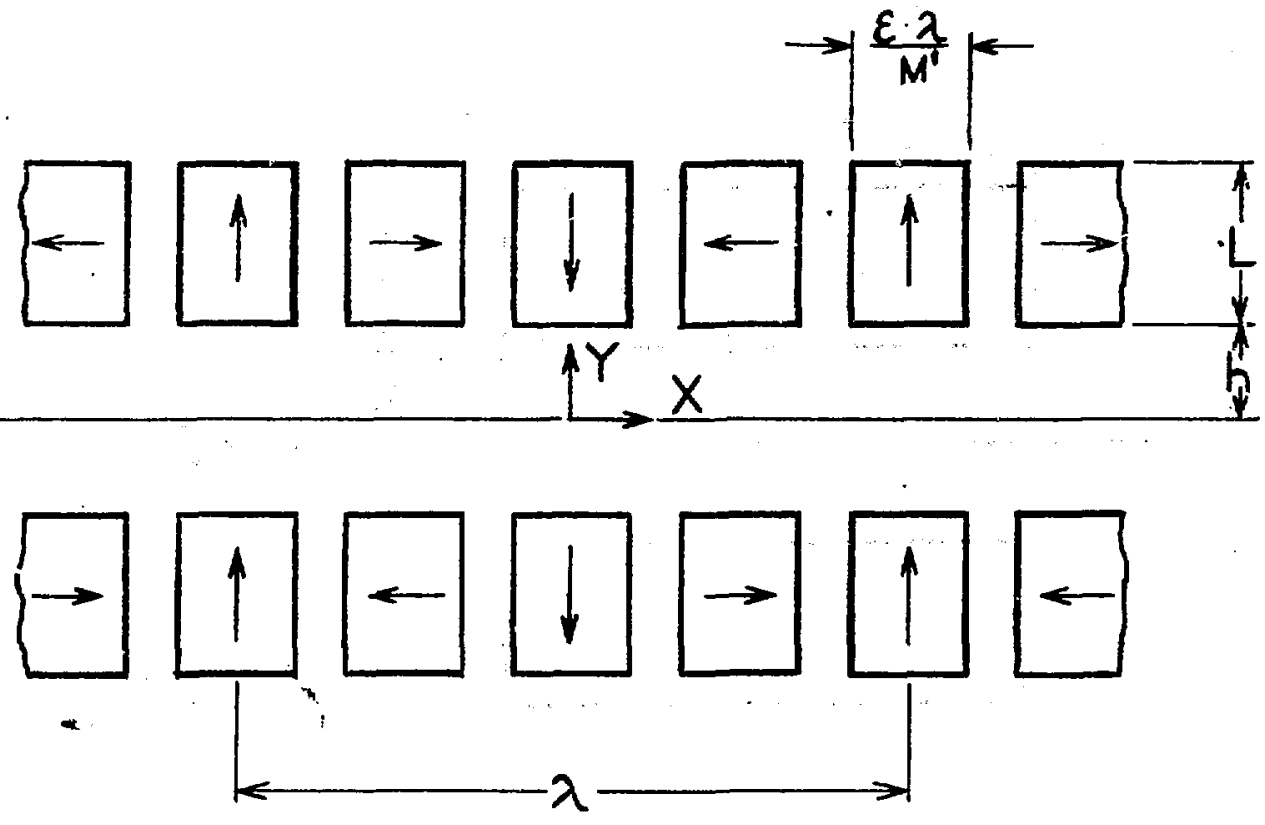

Figure 6 

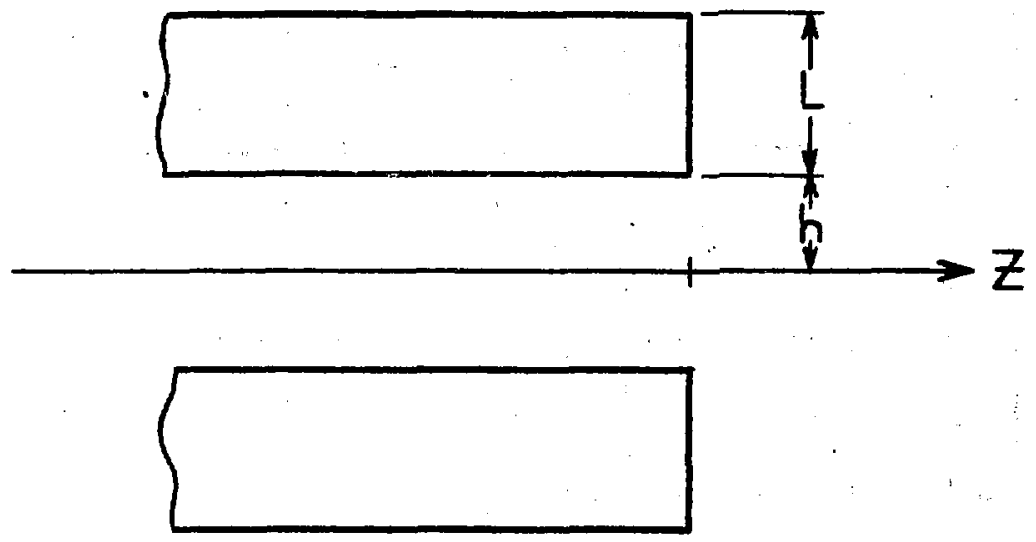

Figure 7 


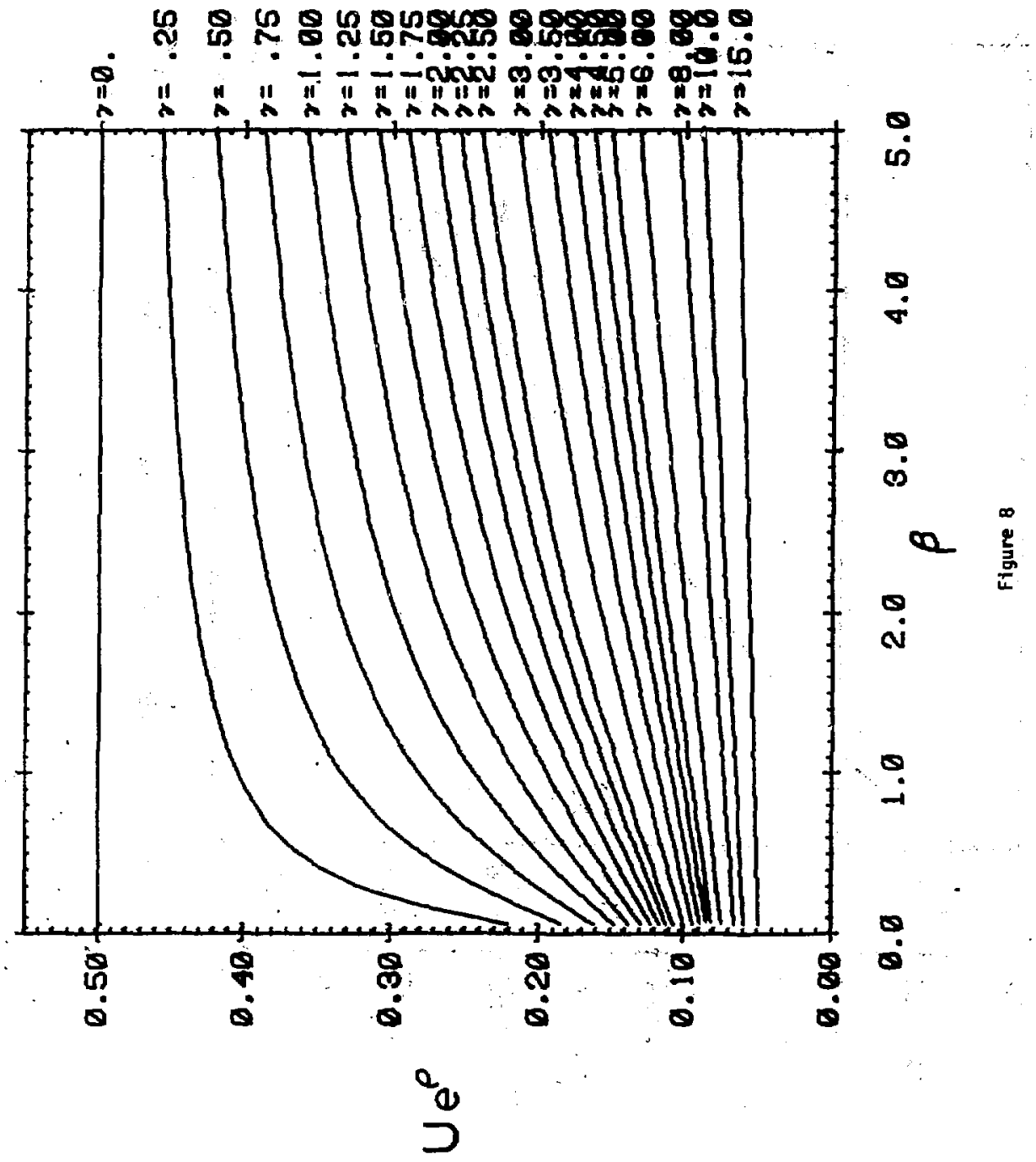




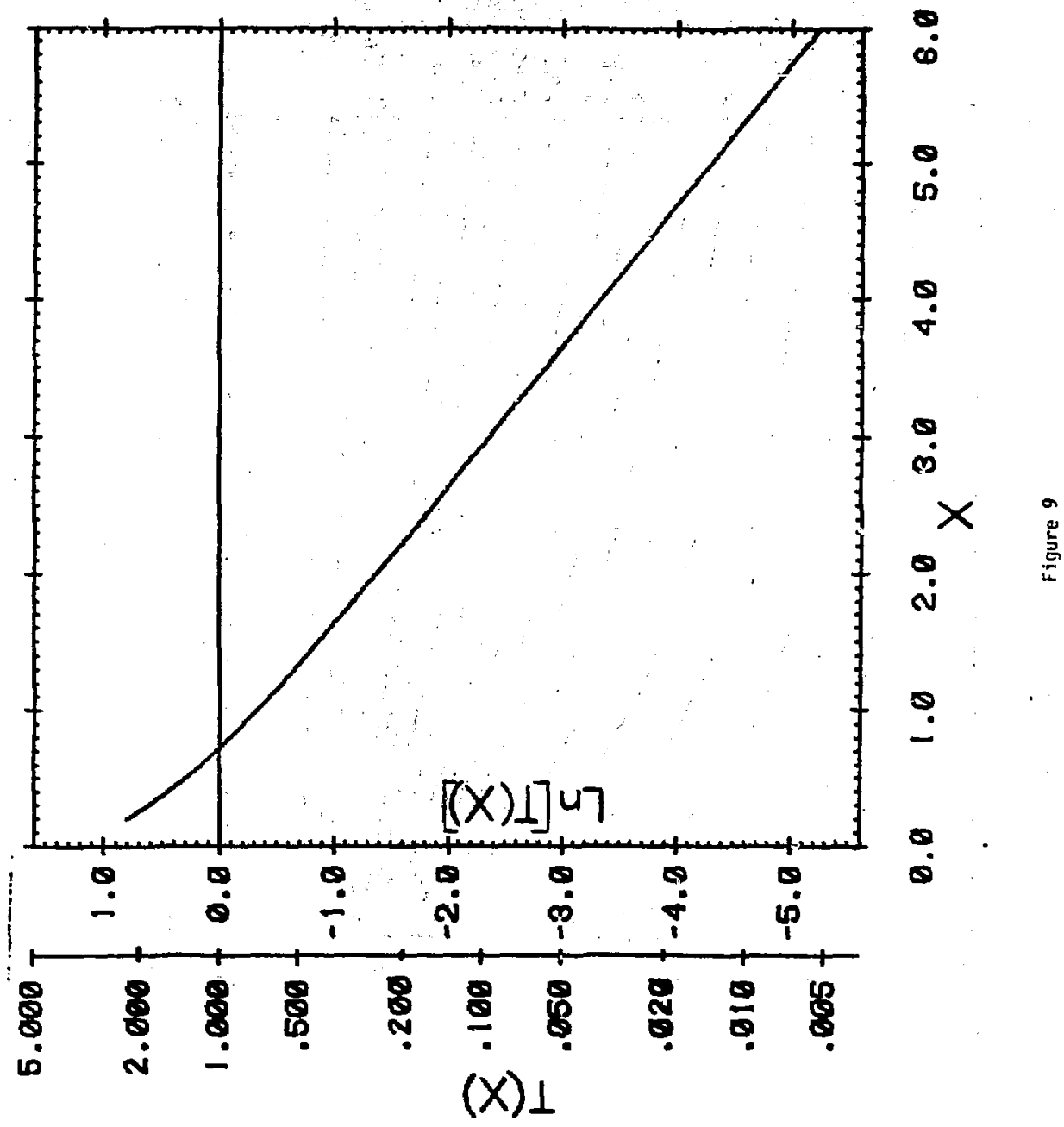



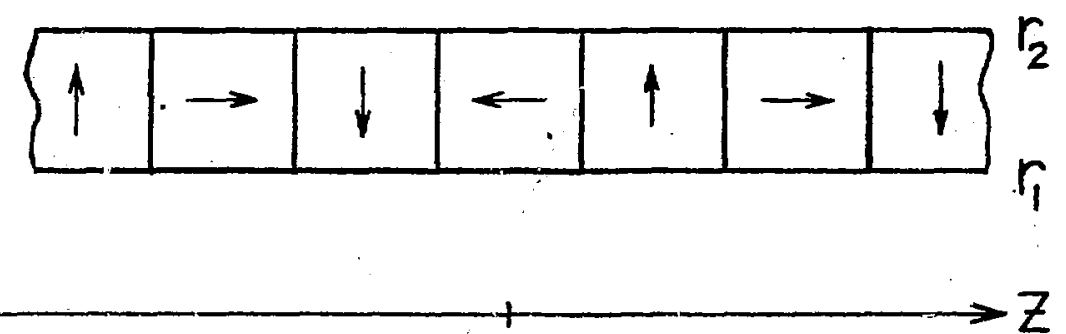

$\rightarrow Z$

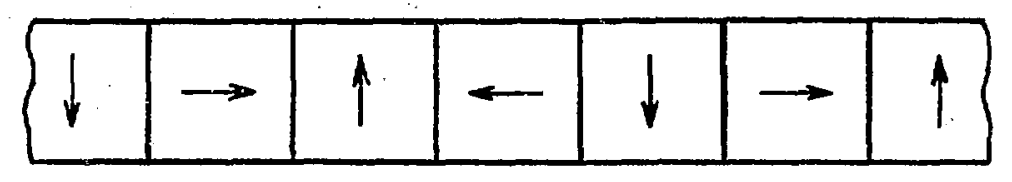

Figure 10 


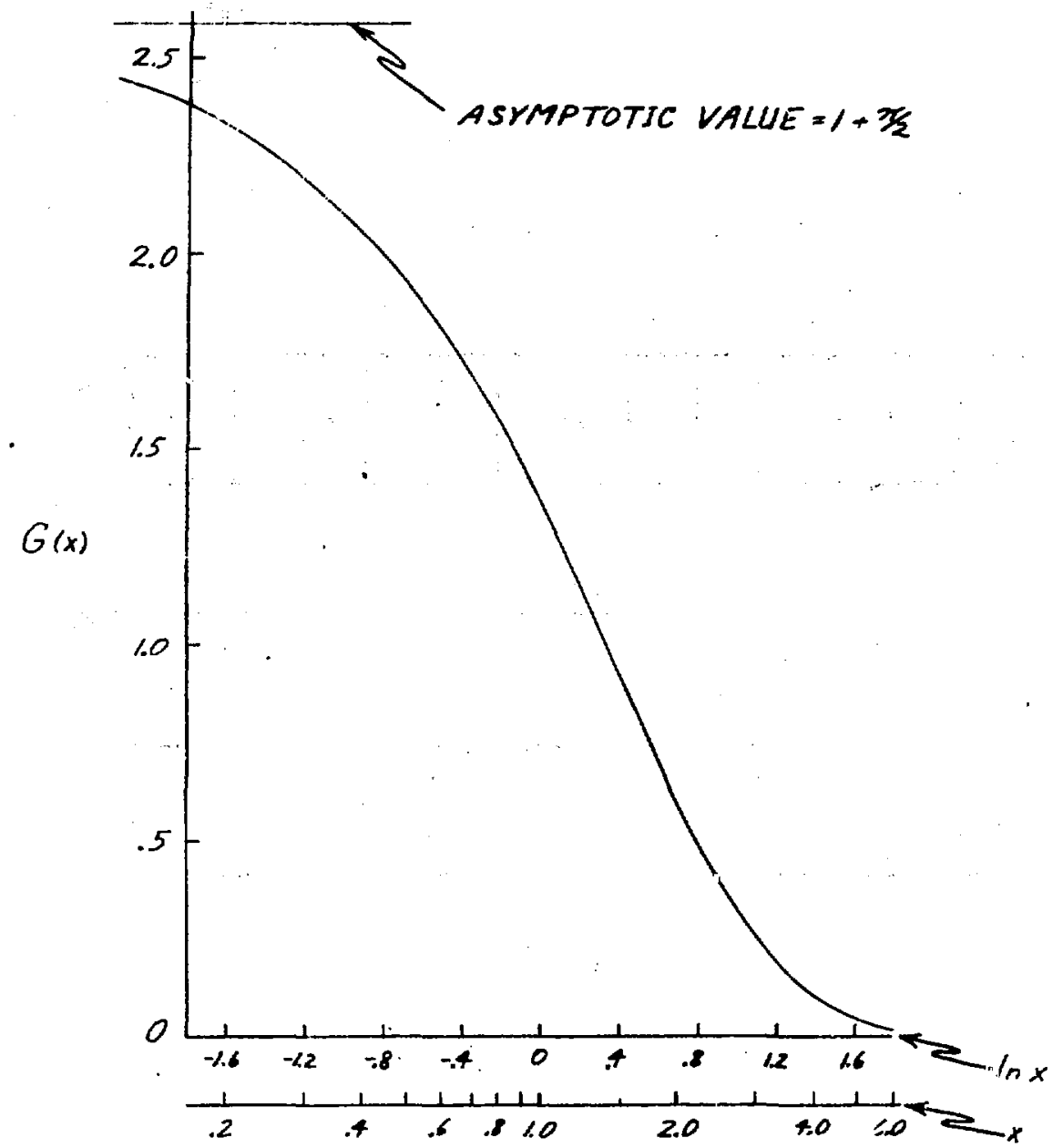

XBL 808-11001

Figure 11 